\title{
Pharmacoeconomic Rationale of Zinc Supplementation In The Management of Acute Diarrhea In Children With Rotavirus Infection In Indonesia
}

\author{
Mohamad S. Hakim ${ }^{1,2,}{ }^{*}$, Serhii Soloviov ${ }^{3,4}$, Hera Nirwati1,2, Yati Soenarto ${ }^{2,5}$, Iryna Dzyublyk ${ }^{3}$, Maryia \\ Leleka ${ }^{6}$, Viktor Trokhymchuk ${ }^{7}$
}

1. Department of Microbiology, Faculty of Medicine, Public Health and Nursing, Universitas Gadjah Mada, Yogyakarta, Indonesia

2. Pediatric Research Office, Faculty of Medicine, Public Health and Nursing, Universitas Gadjah Mada, Yogyakarta, Indonesia

3. Virology Department, Shupyk National Medical Academy of Postgraduate Education, Kyiv, Ukraine

4. Applied Mathematics Department, National Technical University of Ukraine "Igor Sikorsky Kyiv Polytechnic Institute", Kyiv, Ukraine

5. Department of Child Health, Faculty of Medicine, Public Health and Nursing, Universitas Gadjah Mada, Yogyakarta, Indonesia

6. Department of Pharmaceutical Technology and Biopharmacy, Shupyk National Medical Academy of Postgraduate Education, Kyiv, Ukraine

7. Department of Organizations and Economics of Pharmacy, Shupyk National Medical Academy of Postgraduate Education, Kyiv, Ukraine

\author{
Info Article \\ Submitted: 07-03-2019 \\ Revised: 28-09-2019 \\ Accepted: $15-10-2019$ \\ *Corresponding author \\ Mohamad S. Hakim \\ Email: \\ m.s.hakim@ugm.ac.id.
}

\begin{abstract}
Rotavirus is one of the most important causative agents of gastroenteritis in both infants and children worldwide, resulting in a high mortality and burden of disease, mainly in low-income, developing countries. Oral rehydration therapy with zinc supplementation is currently the recommended way to prevent death from diarrheal dehydration. In this study, we aimed to estimate the effectiveness of zinc supplementation in combination therapy of patients with suspected rotavirus infection in Indonesia. The perspective of this pharmacoeconomic study refer to the economic vantage point of cost-of-illness analysis. Achievement of this goal was possible with the construction of a decision tree model and determination of decision rules for inclusion of zinc supplementation into the combination therapy. The input parameters of the model were hospitalization days of patients stratified by the presence or absence of rotavirus infection as well as the additional inclusion of zinc supplementation. The criterion for prediction and decision making was the global rotavirus prevalence. The feature of the simulation was that the costs were expressed as relative to each other, which allowed to unify the proposed methodology. Retrospective analysis of clinical database of Indonesian patients with acute diarrhea has shown that zinc supplementation would be rational in case of rotavirus prevalence among these patients is higher than $81.5 \%$. It was shown that additional zinc supplementation would be costeffective with probability of 0.62 and 0.53 - in the positive range of cost ratio variation or if costs for less than $43 \%$ of baseline therapy per day costs on an average.
\end{abstract}

Keywords: Accute diarrhea, zinc supplementation, rotavirus, infection, pharmaco economic

\section{INTRODUCTION}

The problem of the global spread of acute intestinal infections (AII) is currently relevant, as they occupy one of the leading places among infectious diseases, yielding only the frequency of influenza and acute respiratory illness (GBD 2015 
Mortality and Causes of Death Collaborators, 2016). Among AII, rotavirus infection (RVI) remains the leading cause of morbidity and mortality in children under five years of age, despite a reduction in the number of cases of hospitalization associated with diarrhea and death (Lanata et al., 2013). Despite the fact that the number of deaths from rotavirus gastroenteritis declined from 528,000 in 2000 to 215,000 in 2013, of which over $80 \%$ were registered in countries of Asia and Africa, RVI continues to cause significant damage to public health of all countries regardless of the level of economic development. RVI causes direct and indirect economic costs, estimated at hundreds of billions of dollars per year (Tate et al., 2016; Wazny K et al., 2013).

In Asia, rotavirus was responsible for 145,000 deaths every year, with the highest numbers were found in India, Pakistan and Indonesia (Kawai et al., 2012; Hakim et al., 2018). Years of national surveillance of acute gastroenteritis in Indonesia has demonstrated that rotaviruses are responsible for about $50-60 \%$ cases of acute diarrhea in children less than five years of age (Soenarto et al. 2009; Nirwati et al., 2016; Wilopo et al., 2009). Rotavirus-infected children were more likely to experience severe clinical symptoms, such as vomiting and dehydration (Unger et al., 2014; Miller, 1997; Goel et al., 2010). After establishing infection, rotavirus causes severe diarrhea and vomiting, leading to significant loss of bodily fluids and resulting in dehydration. If unable to rehydrate soon after the symptom onset, the children progress into severe complications, leading to mortality. The greatest advancement in the treatment of diarrhea in children has been achieved through the introduction of World Health Organization (WHO) recommendations, oral rehydration protocols. However, these treatment regimens are aimed only at dehydration correction and do not reduce the frequency, volume of emptying or duration of diarrhea (Sato and Zouain, 2010). The current treatment of acute diarrhea is the inclusion of zinc supplementation, which was recommended by WHO and The United Nations International Children's Emergency Fund (UNICEF) in 2004. According to a number of randomized clinical trials, it has been established that the administration of zinc during diarrhea and several days after the onset of acute gastroenteritis not only reduces the duration and severity of diarrhea, but also prevents the emergence of further cases of gastroenteritis for at least several months (Walker et al., 2010; Roy et al., 2018).
The search for highly effective, safe and relatively inexpensive technologies of pharmacotherapy remains extremely relevant, based on pharmacoeconomic principles, which will reduce the burden on state or regional healthcare budgets. In practice, it is usually necessary to decide on the choice between different technologies of pharmacotherapy, aimed at both the normalization of individual clinical and laboratory indicators of the infectious disease, and its possible cause. In the case of the use of a pharmaceutical drug in the scheme of pharmacotherapy of an infectious disease that can be caused by various pathogens, including viruses, such as acute gastroenteritis, the question arises the development of criteria for its effectiveness, the answer to which can be found with the analysis of retrospective laboratory and pharmacotherapeutic data to detect the relationship between different signs of an infectious disease, the choice of pharmacotherapy and its outcome. Therefore, the aim of our study was to investigate the pahrmacoeconomic rationale of zinc supplementation in the management of acute diarrhea in children with rotavirus infection in Indonesia with the use of analytical modeling.

\section{MATERIALS AND METHODS Clinical data}

In our study, we used clinical dataset, consists of 983 children medical records less than five years of age enrolled from January to December 2010 hospitalized due to acute diarrhea at six hospitals in Indonesia. The participating hospitals were: (1) Muhammad Hussein Hospital (Palembang, South Sumatera); (2) Cipto Mangunkusumo Hospital (Jakarta, Capital City Special Territory); (3) Hasan Sadikin Hopsital (Bandung, West Java); (4) Sardjito Hospital (Yogyakarta, Yogyakarta Special Territory Province); (5) Sanglah Hospital (Denpasar, Bali); and (6) Mataram Hospital (Mataram, West Nusa Tenggara). A standardized clinical form was completed to obtain information on the date of admission, age and sex of the patients, clinical symptoms and final laboratory-confirmed diagnosis.

\section{Treatment outcome evaluation}

The perspective of this pharmacoeconomic study refer to the economic vantage point of cost-of-illness analysis. This affects all types of costs (resource expenditures) and their minimization due to the proposed pharmacotherapy. Pharmaco-economic valuation defines 


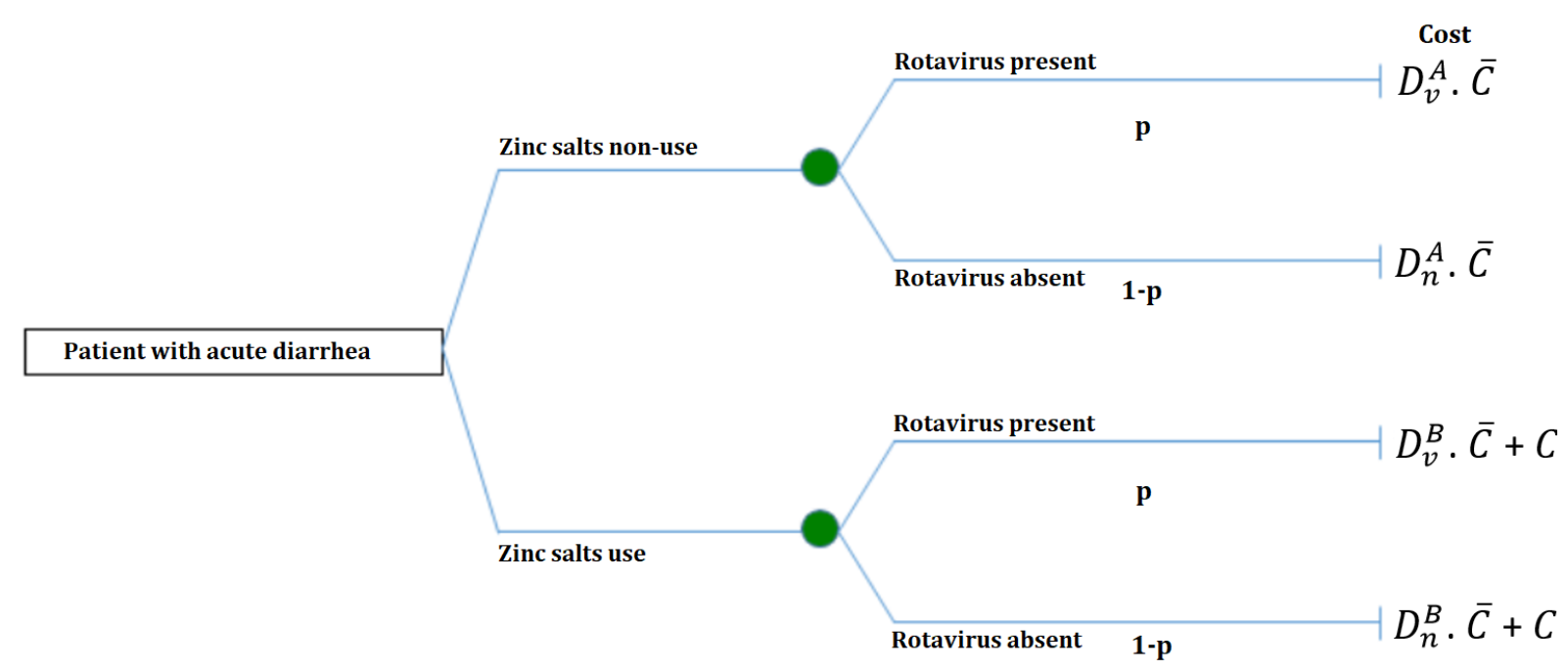

Figure 1. Decision tree of zinc preparation use for patient with acute diarrhea and expected costs

the currency reference that represents the resource expenditure associated with a given costs. However, the costs were expressed as relative to each other, which enable to unify the proposed methodology. Unified methodology could be focused on institutional and patient perspective and treatment costs would be relevant to resource expenditures involved in the analysed therapy.

In the absence of information on the presence of a viral agent, the question arises of the choice of pharmacotherapy for an infectious disease. The algorithm of such a choice depends on possible outcomes: total cost in the absence of a viral infection of interest $\left(C_{n}\right)$, and $\left(C_{v}\right)$ - if present, when choosing the appropriate technology of pharmacotherapy. In order to facilitate the adoption of pharmacotherapeutic solutions, the concept of the maximum probability of the presence of a viral infection $p$ is launched. The decision to choose the scheme of pharmacotherapy is taken in accordance with the maximum probability of the presence of a viral infection (Soloviov et al., 2016). Expected costs $\left(E C^{A}(p)\right)$ when choosing a technology of pharmacotherapy of acute diarrhea without the use of zinc salts (pharmacotherapy A) are determined on the basis of the constructed decision tree (Figure 1).

$E C_{-} A=p D_{-} v^{\wedge} A^{-} \mathrm{C}+(1-p) D_{n}^{A} \overline{\mathrm{C}}=\left[p D_{-} v^{\wedge} A+\right.$ $\left.(1-p) D \_n^{\wedge} A\right]^{-} \mathrm{C}=D_{-} A^{-} \mathrm{C}$

where D - duration of hospital stay, presence (n) or absence (v) of the pathogen, $\bar{C}$ - average costs per one day of baseline therapy.
Expected costs $\left(E C^{B}(p)\right)$ in the choice of alternative pharmacotherapy with the inclusion of zinc salts (pharmacotherapy B)) can be determined from the lower branch of the decision tree as a weighted loss for two cases (rotavirus infection is absent or present), taking into account the relevant probability of their occurrence (Figure 1):

$E \mathrm{C}_{B}=p\left(D_{v}^{B} \overline{\mathrm{C}}+\mathrm{C}_{\mathrm{T}}\right)+(1-p)\left(D_{n}^{B} \overline{\mathrm{C}}+\mathrm{C}_{\mathrm{T}}\right)=$ $\left[p D_{v}^{B}+(1-p) D_{n}^{B}\right] \overline{\mathrm{C}}+\mathrm{C}_{\mathrm{T}}=D_{B} \overline{\mathrm{C}}+\mathrm{C}_{\mathrm{T}}$

where D - duration of hospital stay, presence (n) or absence $(v)$ of the pathogen, $\bar{C}$ - average costs of one day of baseline therapy, CT - costs for additional therapy.

Define the conditions for a comparative choice of each of the four alternatives in relation to others. The technology of pharmacotherapy $B$ is dominant in relation to pharmacotherapy A, if the relevant condition is fulfilled:

$E \mathrm{C}_{B}<E \mathrm{C}_{A}$

$D_{B} \bar{C}+\mathrm{C}_{\mathrm{T}}<D_{A} \bar{C}$

$\frac{C_{\mathrm{T}}}{\bar{C}}<D_{A}-D_{B}$

where:

$D_{A}=\left[p D_{v}^{A}+(1-p) D_{n}^{A}\right]$.

$D_{B}=\left[p D_{v}^{B}+(1-p) D_{n}^{B}\right]+C_{T}$

In general, the expected loss in decision making depends on the value of the prevalence of viral infection $p$. This clearly indicates that it is necessary to refrain from the choice of pharmacotherapy B at a low level of the prevalence of the virus infection and vice versa - at high level. Consequently, this indicates that there should be an intermediate rate of prevalence, in which any 
choice of pharmacotherapy will give the same expected result. This value is the limiting level of the prevalence of viral infection $\tilde{p}$ (Figure 2).

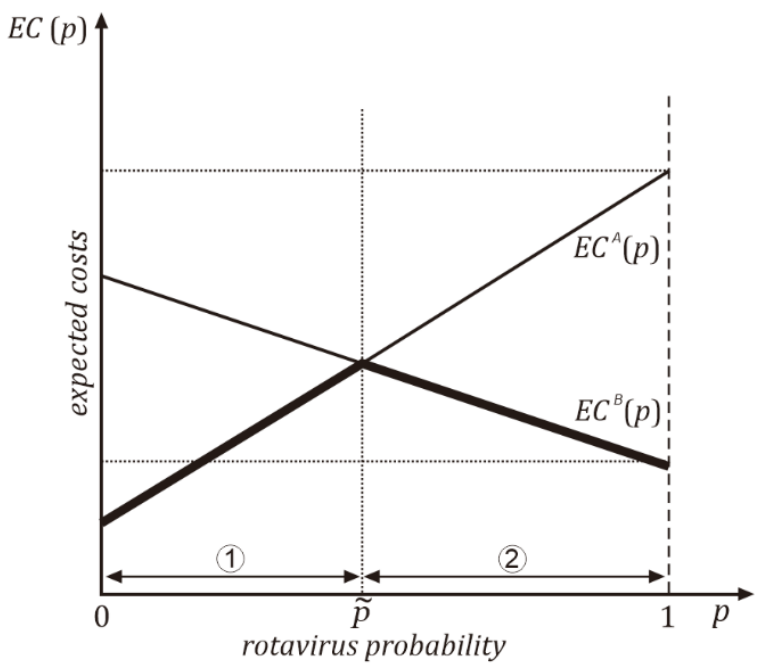

Figure 2. Graphical definition of the marginal level of the prevalence of viral infection as a criterion for decision-making $(1$ - domination of pharmacotherapy without zinc salts, 2 - domination of pharmacotherapy with zinc salts).

The marginal level of prevalence of rotavirus infection is such an extent of prevalence in which any choice of pharmacotherapy leads to the same expected costs $E C^{B}(p)=E C^{A}(p)$, that is:

$\left[p D_{v}^{B}+(1-p) D_{n}^{B}\right] \bar{C}+C_{\mathrm{T}}=\left[p D_{v}^{A}+(1-p) D_{n}^{A}\right] \bar{C}$

The solution of this equation in relation to $p$ gives an expression for determining the extreme level of the prevalence of a viral infection as a criterion for making decisions in the pharmacotherapy of acute diarrhea under conditions of incomplete information:

$\tilde{p}=\frac{D_{n}^{A}-D_{n}^{B}}{D_{v}^{B}-D_{n}^{B}+D_{n}^{A}-D_{v}^{A}}+\frac{1}{D_{v}^{B}-D_{n}^{B}+D_{n}^{A}-D_{v}^{A}} \frac{\mathrm{C}_{\mathrm{T}}}{\bar{C}}$

For the prevalence of rotavirus infection below the marginal, the expected costs from the choice of pharmacotherapy A is less than the corresponding expected costs from the choice of pharmacotherapy B in the range of prevalence $0 \leq$ $p \leq \tilde{p}$. With an a priori increase in the likelihood of a viral infection, the expected loss in the choice of pharmacotherapy $\mathrm{B}$ is reduced while the expected loss in choosing a pharmacotherapy A increases. For higher prevalence rates, the expected loss in

the choice of pharmacotherapy $B$ is less than the corresponding expected loss in the choice of pharmacotherapy A that is dominant in the range of prevalence $\tilde{p} \leq p \leq 1$.

In practice, we often deal with significant variability in the length of hospitalization, which should also be taken into account when the modeling utilize real retrospective clinical and laboratory data. Other assumptions underpinning the decision tree model is the etiological uncertainty of infectious disease within the decision to choose pharmacotherapy. However, if the etiological diagnosis of the viral pathogen is available, previously considered alternative trees can be expanded to include prior etiologic diagnosis followed by pharmacotherapy $\mathrm{B}$.

\section{RESULTS AND DISCUSSION}

Our interest was to study pharmacoeconomic effect of zinc supplementation in patients with or without rotavirus (RV) infection. For every studied clinical dataset, we divided into four smaller datasets according to rotavirus infection presence or absence and zinc supplementation or no. Thus, we were able to divide every clinical dataset into four smaller ones and estimated hospitalization duration distribution (Figure 3) and their mean values (Table I).

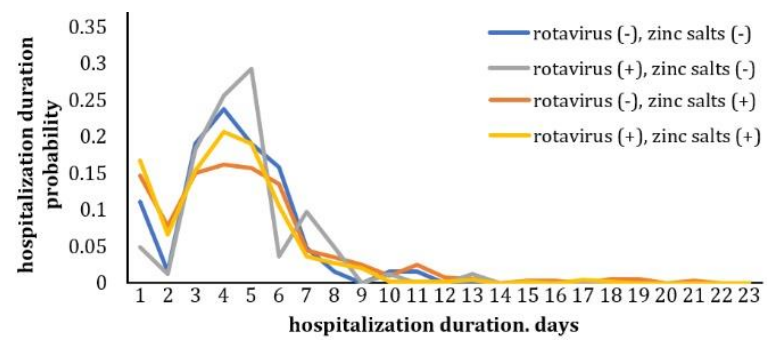

Figure 3. Distribution of hospitalization duration for each alternative.

\begin{tabular}{lcc}
\cline { 1 - 2 } Treatment alternative & $\begin{array}{c}\text { Rotavirus } \\
\text { present }\end{array}$ & $\begin{array}{c}\text { Rotavirus } \\
\text { absent }\end{array}$ \\
\cline { 1 - 2 } Zinc salt use & $D_{v}^{B}=3.230$ & $D_{n}^{B}=3.738$ \\
Zinc salt non-use & $D_{v}^{A}=3.680$ & $D_{n}^{A}=3.365$ \\
\hline
\end{tabular}

Table I. Mean hospitalization duration for each pharmacotherapeutical alternative.

Finding of rotavirus prevalence $(p=0.527)$ from the data set allowed estimation of decision rule for zinc supplementation choice as costs for additional treatment $\left(C_{T}\right) /$ baseline therapy per day costs $(\overline{\mathrm{C}})$ ratio. 


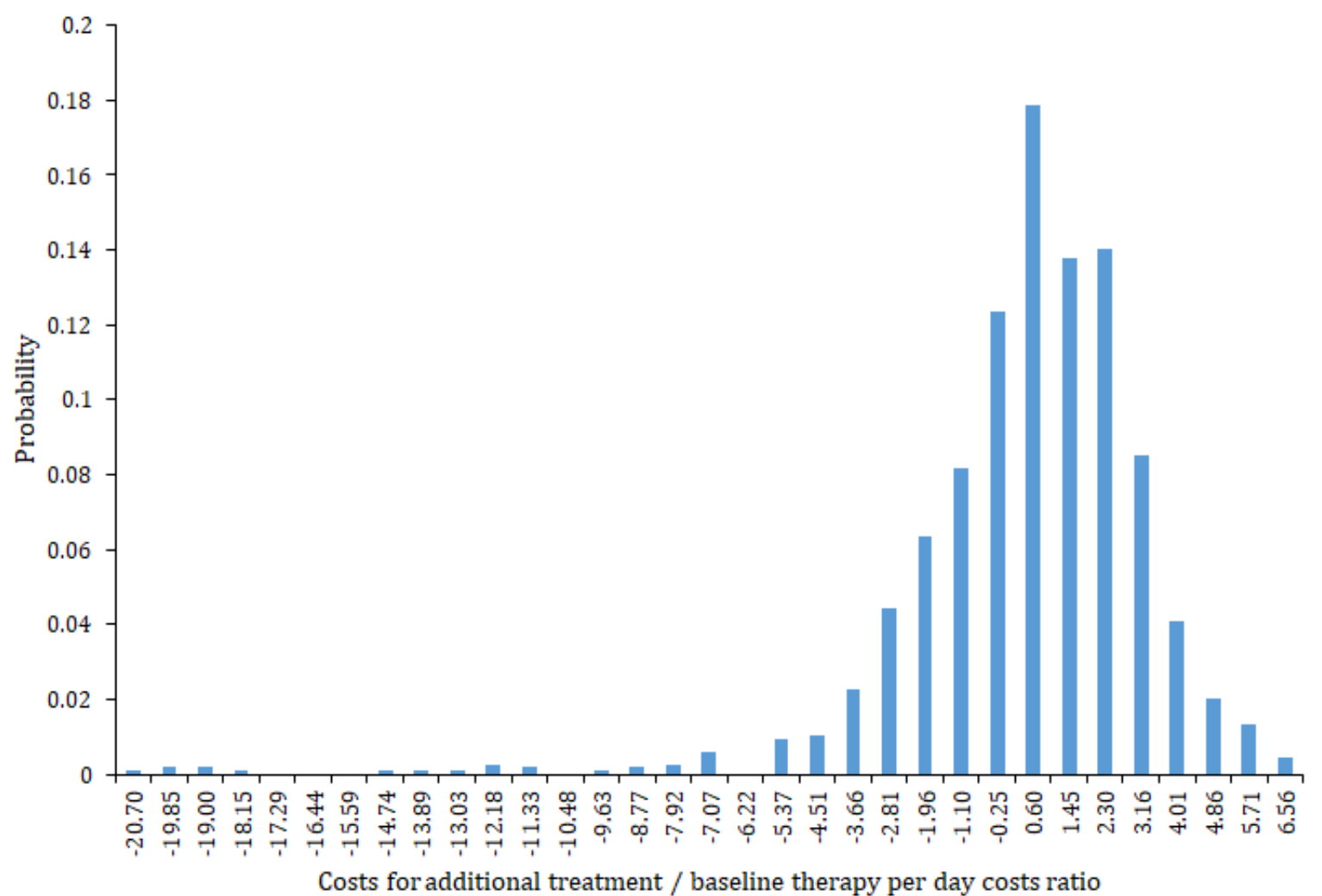

Figure 4. Distribution of costs for additional treatment $\left(C_{T}\right)$ / baseline therapy per day costs $(\bar{C})$ ratio.

Our proposal was to estimate its stochastic value according to formula 3 with the use of previously estimated distributions of hospitalization duration in each of four datasets by simulations in Microsoft Excel (Figure 4). We found that with probability of 0.38 (or $38 \%$ ) this ratio would be in negative values, meaning absolute domination of no zinc supplementation. Nevertheless, for $62 \%$ of simulated results critical ratio existed destinating the maximum value of 6.56 and the mean value 0.43 . It means that in average, decision of zinc supplementation as additional treatment could be appropriate if costs for additional treatment $\left(C_{T}\right)$ would be less than $43 \%$ of baseline therapy per day costs $\bar{C}$. The next step was an analysis of decision criterion - rotavirus prevalence threshold based on formula (7), previously estimated distributions of hospitalization duration in each of four smaller datasets (Figure 3) and randomly distributed $C_{T} / \overline{\mathrm{C}}$ ratio in the real range $[0 ; 6.56]$. Simulation results showed normally distributed values of critical rotavirus prevalence with expected one of 0.815 (Figure 5). This result means that zinc supplementation is justified in case if rotavirus prevalence among patients with acute diarrhea will be more than $81.5 \%$. Negative of more than one values of critical rotavirus prevalence correspond to the dominance of zinc supplementation or non-use, respectively, regardless of rotavirus prevalence level. Comparing this expected value with the real rotavirus prevalence among patients in the clinical database $(p=0.527)$, we found that zinc inclusion into acute diarrhea treatment would be rational from pharmacoeconomic point of view for patients aged less than five years in Indonesia in case of higher rotavirus prevalense rate $(>81.5 \%)$. In addition, we found with the use of simulation that cumulative probability of zinc salts inclusion effectiveness would be of 0.533 (probability of critical rotavirus prevalence less then 0.527), meaning that only for $53.3 \%$ of target patients such inclusion would be cost-effective.

In our work, we studied analyticaly probable cost-effectiveness of zinc supplementation into the management of patients with clinical symptoms of acute diarrhea and suspected rotavirus infection. 


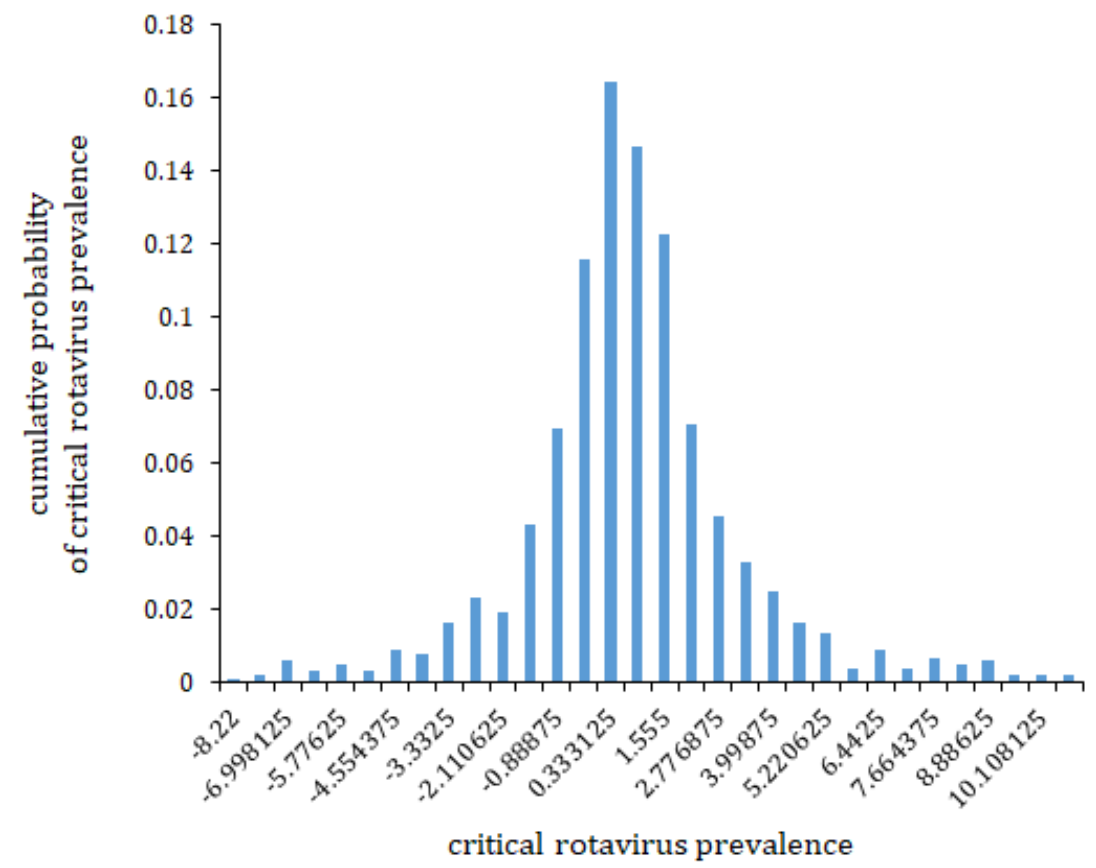

Figure 5. Distribution of critical rotavirus prevalence

The effectiveness of the administration of zinc preparations depends primarily on the age of the children: the more pronounced effect (relative to the duration and severity of the disease) is described in children aged 6-54 months, but the positive effect of zinc compounds and 1-6 month infants has been proven.

In recent years, there has been a widespread discussion in the literature on whether zinc supplementation is effective in diarrhea in patients with zinc deficiency or in patients with normal levels of zinc. Sazawal et al. (1995) reported reduced symptoms of diarrhea in patients receiving zinc supplementation in patients with insufficient zinc level (baseline plasma zinc level was less than $60 \mathrm{~g} / \mathrm{dl}$ ). In experimental studies, it has been shown that the addition of zinc is effective in intestinal infections in patients with normal nutrition and normal initial levels of zinc in the blood (Crane et al., 2007; Kelleher et al., 2002). For the treatment of young children, sulfate, gluconate, or zinc acetate are used, but the special advantages or side effects of each of these zinc salts are not well established. The side effects of the appointment of zinc drugs include disorders of gastrointestinal tolerance, regurgitation, vomiting, but these symptoms in most children are short-term. Vomiting is usually one-time. High doses of zinc have a negative effect on the cells of the immune system, and there are changes similar to those observed with zinc deficiency. In particular, incubation of mononuclear cells with zinc-enriched environments resulted in the release of cytokines interleukin 1 (IL-1), IL-6, tumor necrosis factor $\alpha$ (TNF- $\alpha$ ), soluble IL-2 receptors and interferon- $\gamma$ (IFN- $\gamma$ ) (Prasad, 2008). In our study, we found that zinc supplementation in the management of children with diarrhea results in wide result of uncertainity that could be explained by the fact that the studied dataset consisted of patients of various age groups. E.g., it was showed high probability (0.62) that zinc supplementation was not costeffective and 0.53 - in the positive range of cost ratio variation. The majority of studies showed that zinc supplementation play a significant role in children with acute diarrhoea by accelerating intestinal mucosa regeneration and supporting electrolyte transports. Related to the area of uncertainty, evidences show that zinc is significantly beneficial in children with acute diarrhoea (Crisinel et al., 2015; Patel et al., 2009; Putri et al. 2019).

There are known pharmacoeconomic studies of the use of zinc supplementation in the treatment of acute diarrhea in children. These studies showed the effectiveness and costeffectiveness of such additional therapy. The use of zinc with oral rehydration solutions (ORS) reduces 
$5 \%$ of the total cost of treatment and 17 hours of the duration of acute diarrhea. It also reduces $8 \%$ costs of hospitalization and $6 \%$ costs of antibiotics in the supplemented group which are then translated into large national savings (Gregorio et al. 2007; Patel et al., 2003). Nevertheless, these studies focused on cost-effectiveness analysis involving evaluation of effectiveness of zinc supplementation which is difficult in case of acute diseases. We went further in this regard to pharmacoeconomic assessment, not only using only costs, but also presenting them in a relative unified form, which allows us to use this methodology in different financial conditions. Definitely, our study has some limitations. In our study, we only estimated zinc supplementation in combination treatment of acute diarrhea. Nevertheless, it can also be performed easily of any combination of treatment effectiveness. However, from the other side, this is a huge analytical work, which is beyond the scope of this paper.

\section{CONCLUSIONS}

Inclusion of zinc supplementation is a new tool in the treatment protocols of acute diarrhea in children. This effective and inexpensive treatment not only reduces the incidence of severe gastroenteritis, but also prevents the following cases of diarrhea among children. The WHO comprehensive strategy, which includes both known methods (breastfeeding, careful compliance with hygiene), and new principles - modified recipes for solutions for oral rehydration, vaccination against rotavirus, the administration of zinc preparations, can become an effective method for reducing diseases, which are accompanied by diarrhea, both in developing countries and in developed countries.

Oral rehydration therapy with zinc supplementation is currently the recommended way to prevent death from diarrheal dehydration. Estimation of zinc supplementation effectiveness in combination therapy of patients with suspected rotavirus infection in Indonesia was possible with the construction of a decision tree model and determination of decision rules for inclusion of zinc salts into the combination therapy. Retrospective analysis of clinical database of Indonesian patients with acute diarrhea has shown that zinc supplementation would be rational in case of rotavirus prevalence among these patients is higher than $81.5 \%$ or costs for additional treatment (zinc supplementation) would be less then $43 \%$ of baseline therapy per day costs.

\section{ACKNOWLEDGEMENT}

MSH is supported by the Indonesia Endowment Fund for Education (LPDP). We thank the parents of all children for their willingness to participate.

\section{REFERENCES}

Crane J., Naeher T., Shulgina I., Zhu C. and Boedeker E. 2007. Effect of zinc in enteropathogenic Escherichia coli infection. Infect Immun. 75(12): 5974-5984.

Crisinel PA., Verga M., Kouame KSA., Pittet A., ReyBellet CG., et al. 2015. Demonstration of the effectiveness of zinc in diarrhoea of children living in Switzerland. Eur J Pediatr. 174: 1061-1067.

GBD 2015 Mortality and Causes of Death Collaborators. 2016. Global, regional, and national life expectancy, all-cause mortality, and cause-specific mortality for 249 causes of death, 1980-2015: a systematic analysis for the Global Burden of Disease Study 2015. Lancet. 388: 1459-1544.

Goel MK., Khanna P. and Kishore J. 2010. Understanding survival analysis: KaplanMeier estimate. Int J Ayurveda Res. 1(4): 274278.

Gregorio G.V, Dans LF, Cordero CP, Panelo CA. 2007. Zinc supplementation reduced cost and duration of acute diarrhea in children. J Clin Epidemiol. 60(6): 560-566.

Hakim MS., Nirwati H., Aman AT., Soenarto Y. and Pan Q. 2018. Significance of continuous rotavirus and norovirus surveillance in Indonesia. World J Pediatr. 14(1): 4-12.

Kawai K., O'Brien MA., Goveia MG., Mast TC. and El Khoury AC. 2012. Burden of rotavirus gastroenteritis and distribution of rotavirus strains in Asia: a systematic review. Vaccine. 30:1244-1254.

Kelleher S., Casa I., Carbajal N. and Lonnerdal B. 2002. Supplementation of infant formula with the probiotic Lactobacillus reuteri and zinc: impact on enteric infection and nutrition in infant rhesus monkeys. J Pediatr Gastroenterol Nutr. 35: 162-168.

Lanata CF., Fischer-Walker CL., Olascoaga AC., Torres CX., Aryee MJ., et al. 2013. Child Health Epidemiology Reference Group of the World Health Organization and UNICEF. Global causes of diarrheal disease mortality in children $<5$ years of age: a systematic review. PloS One. 8:e72788 
Miller RG. Survival analysis. John Wiley \& Sons 1997.

Nirwati H., Wibawa T., Aman AT., Wahab A. and Soenarto Y. 2016. Detection of group A rotavirus strains circulating among children with acute diarrhea in Indonesia. Springerplus. 5: 97.

Patel A., Dibley MJ., Mamtani M., Badhoniya N. and Kulkarni H. 2009. Zinc and copper supplementation in acute diarrhoea in children: a double-blind randomized controlled trial. BMC Medicine. 7: 22.

Patel AB., Dhande LA. and Rawat MS. 2003. Economic evaluation of zinc and copper use in treating acute diarrhea in children: A randomized controlled trial. Cost Eff Resour Alloc. 1(1): 7.

Prasad A. 2008. Zinc in human health: effect of zinc on immune cells. Mol Med. 14(5-6): 353357.

Putri W., Akhmad S. and Desrini S. 2019. The role of zinc supplementation for diarrhoea in children: a critical review. Bangladesh Journal of Medical Science. 18(2), 190-195.

Roy SK., Hossain MJ., Khatun W., Chakraborty B., Chowdhury S., et al. 2008. Zinc supplementation in children with cholera in Bangladesh: randomised controlled trial. BMJ. 336(7638): 266-268.

Sato RC. and Zouain DM. "Markov Models in health care." Einstein (São Paulo) 8.3. 2010.

Sazawal S., Black RE., Bhan MK., Bhandari N., Sinha A., et al. 1995. Zinc supplementation in young children with acute diarrhea in India. N Engl J Med. 333: 839-844.
Soenarto Y., Aman AT., Bakri A., Waluya H., Firmansyah A., et al. 2009. Burden of severe rotavirus diarrhea in Indonesia. J Infect Dis. 200 Suppl 1: S188-194.

Soloviov SO., Dzyublyk IV., Zaliska OM. and Sakhno GO. 2016. Analytical substantiation of the inclusion of an antiviral drug in the treatment of patients with suspected acute viral diseases. Annals of the Mechnikovsky Institute. 4: 18-26.

Tate JE., Burton AH., Boschi-Pinto C., Parashar UD; 2016. World Health OrganizationCoordinated Global Rotavirus Surveillance Network.. Global, regional, and national estimates of rotavirus mortality in children $<5$ years of age, 2000-2013. Clin Infect Dis. 62(Suppl 2): S96-S105.

Unger CC., Salam SS., Sarker MSA., Black R., Cravioto A., et al. 2014. Treating diarrheal disease in children under five: the global picture. Archives of Disease in Childhood. 99: 273.

Walker C., Black R. 2010. Zinc for the treatment of diarrhea: effect on diarrhea morbidity, mortality and incidence of future episodes. Intern J Epidemiology. 39: 63-69.

Wazny K., Zipursky A., Black R., Curtis V., Duggan C., et al. 2013. Setting research priorities to reduce mortality and morbidity of childhood diarrhoeal disease in the next 15 Years. PLoS Med. 10(5): e1001446.

Wilopo SA., Soenarto Y., Bresee JS., Tholib A., Aminah S., et al. 2009. Rotavirus surveillance to determine disease burden and epidemiology in Java, Indonesia, August 2001 through April 2004. Vaccine. 27 (Suppl 5): F61-66. 